\title{
Follow-Up of Liver Stiffness with Shear Wave Elastography in Chronic Hepatitis C Patients in Sustained Virological Response Augments Clinical Risk Assessment
}

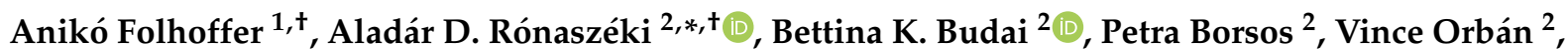 \\ Gabriella Győri ${ }^{2}$, Ferenc Szalay ${ }^{1}$ and Pál N. Kaposi ${ }^{2}$ (D)
}

1 Department of Internal Medicine and Oncology, Faculty of Medicine, Semmelweis University, Korányi S. u. 2/a, H-1083 Budapest, Hungary; folhoffer.aniko@med.semmelweis-univ.hu (A.F.); szalay.ferenc@med.semmelweis-univ.hu (F.S.)

2 Department of Radiology, Medical Imaging Centre, Faculty of Medicine, Semmelweis University, Korányi S. u. 2., H-1083 Budapest, Hungary; budai.bettina@med.semmelweis-univ.hu (B.K.B.); petraborsos96@gmail.com (P.B.); orban.vince@med.semmelweis-univ.hu (V.O.); gyori.gabriella@med.semmelweis-univ.hu (G.G.); kaposi.pal@med.semmelweis-univ.hu (P.N.K.)

* Correspondence: ronaszeki.aladar_david@med.semmelweis-univ.hu; Tel./Fax: +36-(1)-459-1500 x.61626

+ These authors contributed equally to this work.

\section{check for}

updates

Citation: Folhoffer, A.; Rónaszéki, A.D.; Budai, B.K.; Borsos, P.; Orbán, V.; Győri, G.; Szalay, F.; Kaposi, P.N. Follow-Up of Liver Stiffness with Shear Wave Elastography in Chronic Hepatitis C Patients in Sustained Virological Response Augments Clinical Risk Assessment. Processes 2021, 9, 753. https://doi.org/ $10.3390 /$ pr9050753

Academic Editor: Katarzyna Otulak-Kozieł

Received: 30 March 2021

Accepted: 22 April 2021

Published: 24 April 2021

Publisher's Note: MDPI stays neutral with regard to jurisdictional claims in published maps and institutional affiliations.

Copyright: (c) 2021 by the authors. Licensee MDPI, Basel, Switzerland. This article is an open access article distributed under the terms and conditions of the Creative Commons Attribution (CC BY) license (https:// creativecommons.org/licenses/by/ $4.0 /)$.
Abstract: This study aimed to observe the effect of the direct-acting antiviral (DAA) therapy on liver stiffness (LS) and serum biomarkers. We prospectively observed 35 patients with chronic hepatitis $C$ infection and attained a sustained virological response (SVR) after antiviral therapy. Shear wave elastography (SWE) measurement was performed at the beginning of DAA treatment and at 48 weeks after the end of treatment (EOT48w). The METAVIR score and the score for varices needing treatment (VNT) were determined based on the LS values; the fibrosis-4 (FIB4) score was calculated from laboratory tests. The baseline LS (mean \pm standard deviation $=2.59 \pm 0.89 \mathrm{~m} / \mathrm{s}$ ) decreased significantly after successful DAA therapy $(1.90 \pm 0.50 \mathrm{~m} / \mathrm{s} ; p<0.001)$. The METAVIR score showed significant improvement at EOT48w $(\mathrm{F} 0 / 1=9, \mathrm{~F} 2=2, \mathrm{~F} 3=10, \mathrm{~F} 4=14)$ compared to the initial status $(\mathrm{F} 0 / 1=2, \mathrm{~F} 2=1, \mathrm{~F} 3=7, \mathrm{~F} 4=25 ; p<0.028)$. The FIB4 score indicated less fibrosis after therapy $(2.04 \pm 1.12)$ than at baseline $(3.51 \pm 2.24 ; p<0.018)$. Meanwhile, the number of patients with a high-risk of VNT was significantly less at EOT48w (4 vs. 15 at baseline; OR $=0.1795 \%$ confidence interval $(\mathrm{CI})=0.05-0.59, p<0.007)$. SWE indicates a significant resolution of liver fibrosis when chronic hepatitis $C$ patients are in SVR, coinciding with a lower risk of VNT.

Keywords: chronic hepatitis C; HCV virus; direct-acting antivirals; shear wave elastography; liver stiffness

\section{Introduction}

Hepatitis $\mathrm{C}$ infection (HCV) is a major cause of liver morbidity worldwide, with approximately 71 million chronically infected patients. Due to significant progress in the medical therapy of HCV, the World Health Organization (WHO) is aiming for the elimination of HCV infection as a public health threat by 2030 , achieving a $90 \%$ drop in the incidence compared with 2015 [1]. Interferon (IFN) free direct-acting antivirals (DAAs) have become the standard treatment of HCV hepatitis since 2014 when they were first approved for clinical use by the European Medical Agency [2]. The therapeutic efficiency of DAAs is significantly higher than previous IFN-based regimens, with an eradication rate as high as $90 \%$ in non-cirrhotic patients. The DAAs also have accelerated treatment protocols, usually 12 to 24 weeks, cause fewer side effects, and are associated with better patient compliance compared with IFN [3].

Assessment of liver fibrosis is of high importance prior to therapy. The diagnosis of advanced fibrosis (METAVIR stage F3) and cirrhosis (METAVIR stage F4) can alter 
the choice of a treatment regimen, the therapeutic response rate, and the post-treatment prognosis in affected patients [2,4]. International guidelines now recommend the treatment of all HCV-infected patients and emphasize that those with significant liver fibrosis or cirrhosis must be considered for treatment without delay. Patients with F3 and F4 stage fibrosis also require continued surveillance for HCC every six months, even after successful completion of therapy. Non-invasive methods are preferred over liver biopsy for initial assessment of liver fibrosis in this setting [5,6]. Shear wave elastography (SWE) is a universally accepted non-invasive technique for the staging of fibrosis in chronic hepatitis C $[7,8]$. It measures the liver stiffness (LS) and can be completed in accession to routine ultrasound evaluation of liver morphology. SWE was thoroughly validated by multiple studies and was found to have very good accuracy and reproducibility for the diagnosis of clinically significant fibrosis (METAVIR stage $\geq$ F2) and cirrhosis (F4) $[9,10]$. In addition, SWE does not need specific instrumentation, as this application is commonly available on most newly developed ultrasound scanners, which significantly contributed to the wide-scale acceptance of the technique.

International guidelines universally recommend non-invasive methods for the initial assessment of liver fibrosis in chronic hepatitis $C$ patients. However, we have only limited experience applying elastography for patient follow-up after completing the antiviral treatment [11]. Previous studies mostly used transient elastography (TE) to detect changes in the fibrosis stage in response to INF-based regimens [12,13]. These reports indicated a more substantial decrease in LS of patients with a sustained virological response (SVR) compared to relapsers or non-responders. The short-term improvement in the LS observed at 12 and 24 weeks after the end-of-treatment (EOT) was attributed to the decline in the inflammatory reaction after clearing the HCV infection. Meanwhile, an improved hepatic venous pressure gradient (HVPG) and the resolution of the fibrotic changes on liver biopsy were also reported during long-term follow-up of patients with SVR [14]. Acoustic radiation force impulse (ARFI) elastography, a technique similar to SWE, was used for the follow-up of DAA-treated patients in only a handful of studies, which found significant regression in the fibrosis stage in SVR [15].

There is very little information on the post-treatment follow-up of patients with SWE who have a high-risk of cirrhosis-associated complications. Currently, patients diagnosed pre-treatment with F3 or F4 stage fibrosis need life-long ultrasound surveillance for HCC; however, it is unclear how long the higher risk of HCC persists in SVR and after SWE detects a decrease in fibrosis [16]. Clinically significant HVPG and esophageal varices (EV) are severe complications in compensated advanced chronic liver disease (cACLD) and are also associated with poor disease outcomes [17]. Elastography techniques are able to identify patients with varices needing treatment (VNT) by exposing highly elevated LS values in severe portal hypertension. Consequently, the Baveno VI consensus proposed the assessment of LS and platelet count in its criteria for non-invasive screening for VNTs [18]. Currently, there is limited information available on the risk of VNT after successful antiviral therapy in hepatitis $C$ patients.

The aim of our study was to detect changes in the LS with SWE in chronic hepatitis C patients after treatment with new antiviral protocols. We also aimed to show that SWE can re-evaluate risk factors of cirrhosis-associated complications in SVR.

\section{Materials and Methods}

\subsection{Patient Population}

The present study was approved by the institutional ethics committee of our university (SE-TUKEB 163/2017), and written informed consent was obtained from all subjects according to the World Medical Association Declaration of Helsinki, revised in 2000 in Edinburgh. We prospectively enrolled 35 patients who were diagnosed with chronic HCV infection and who attained SVR after treatment with DAA agents $(n=32)$ or interferonbased treatment $(n=3)$ between October 2016 and January 2020. The inclusion criteria were the follows: patients had to be older than 18 years, the HCV infection had to be 
proved with anti-HCV antibody positivity and HCV RNA positivity by PCR test for at least 6 months prior to the beginning of the antiviral treatment, and the patient had to consent to antiviral therapy and have attained sustained viral response after antiviral therapy. Patients with extrahepatic cholestasis, hepatocellular carcinoma, known congestive heart failure, unsuccessful shear wave elastography measurements, or mixed etiology liver disease were excluded from the study. BMI $>30 \mathrm{~kg} / \mathrm{m}^{2}$, pregnancy, and breastfeeding were also exclusion criteria. The final patient cohort included 19 males and 16 females with a median age of 61 years (interquartile range: 59-65.5 years). All patients underwent a baseline ultrasound scan, which included a liver stiffness measurement with shear wave elastography (SWE) before starting the antiviral treatment. The SWE was repeated in an average of 48 weeks after the end of the antiviral treatment (EOT48w). The patients received the following antiviral combinations: sofosbuvir (SOF)/ledipasvir $(n=5)$, SOF/ledipasvir/ribavirin (RBV) $(n=10)$, ombitasvir / paritaprevir / ritonavir/dasabuvir $(n=15)$, glecaprevir/pibrentasvir $(n=1), \mathrm{SOF} / \operatorname{simeprevir}(n=1)$, peginterferon alpha-2a (PegIFN)/RBV $(n=2)$, and PegIFN/RBV/boceprevir $(n=1)$. The treatment duration was 12 weeks in 21 patients, 24 weeks in 11 patients, and 48 weeks in 3 patients.

\subsection{Liver Stiffness Measurements with Shear Wave Elastography}

A Samsung RS80 Prestige or an RS85A ultrasound scanner (Samsung Medison, Hongcheon, Korea) equipped with the CA1-7A convex probe was used for the liver ultrasound scans. All SWE measurements were performed with the S-Shearwave ${ }^{\mathrm{TM}}$ application following the recommended protocol of the manufacturer. The median LS of at least five measurements was reported in $\mathrm{m} / \mathrm{s}$ units, and it was converted to kilopascal $(\mathrm{kPa})$ to determine the risk of clinically significant hypertension $(>20 \mathrm{kPa})$. Only measurements with a reliable measure index $(\mathrm{RMI}) \geq 0.4$ were considered acceptable. The SWE was technically successful if we were able to collect at least five reliable LS values, and the interquartile range (IQR) of the individual measurements was $<30 \%$ of the median (Figures 1 and 2). The METAVIR score of liver fibrosis was determined for each patient both before and after the antiviral treatment based on the result of the SWE. The following cutoff values were used: F0/1<1.46 m/s, F2 $\geq 1.46 \mathrm{~m} / \mathrm{s}, \mathrm{F} 3 \geq 1.63 \mathrm{~m} / \mathrm{s}, \mathrm{F} 4 \geq 1.95 \mathrm{~m} / \mathrm{s}$, as was described previously [10]. The amount of ascites, if present, was also reported based on the ultrasound scan.

\subsection{Collection of Laboratory Tests and Clinical Data}

Serum creatinine (CRE), sodium (Na), aspartate transaminase (AST), alanine aminotransferase (ALT), gamma-glutamyl transferase (GGT), total bilirubin (TBILL), albumin (ALB) levels, platelet count (PLT), and international normalization ratio (INR) were collected from electronic medical records of the patients at baseline and at the time of the post-treatment SWE. HCV RNA in the serum was detected with an AmpliPrep/COBAS, TaqMan version 2 assay (Roche, Pleasanton, CA, USA). SVR was defined as undetectable HCV RNA at both 24 and 48 weeks after treatment. The model for end-stage liver disease (MELD) and Child-Turcotte-Pugh (CTP) scores and the Fibrosis-4 score for liver scarring (FIB4) were calculated from laboratory parameters with the MDCalc online tool (www.mdcalc.com, accessed on 12th March 2021). Following the recommendations of the Baveno VI guidelines, the patients were also divided into low-risk and high-risk (PLT < $150 \mathrm{G} / \mathrm{L}, \mathrm{LS}>20 \mathrm{kPa}$ ) groups of esophageal VNT [17] (Figure 3). 


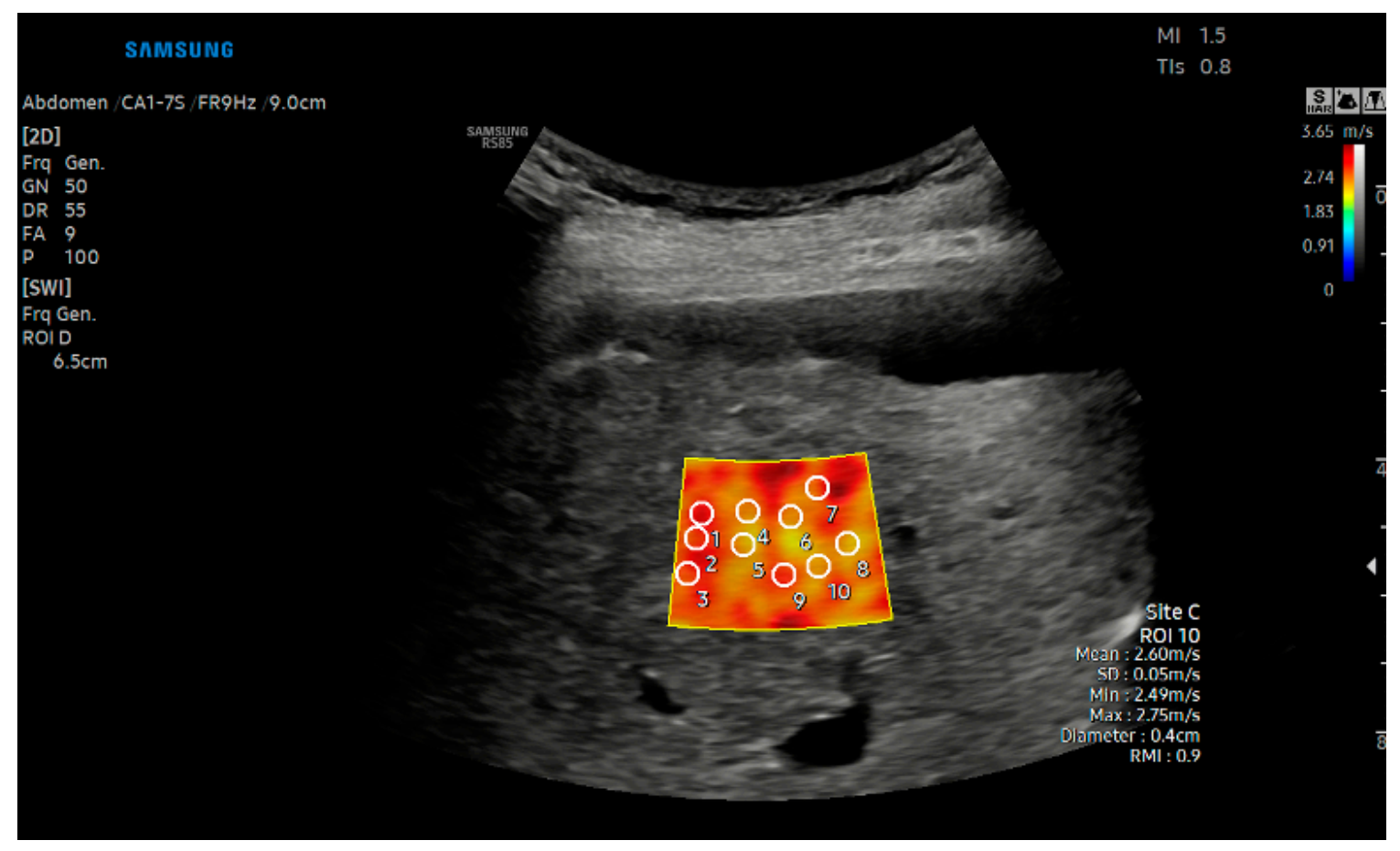

(a)

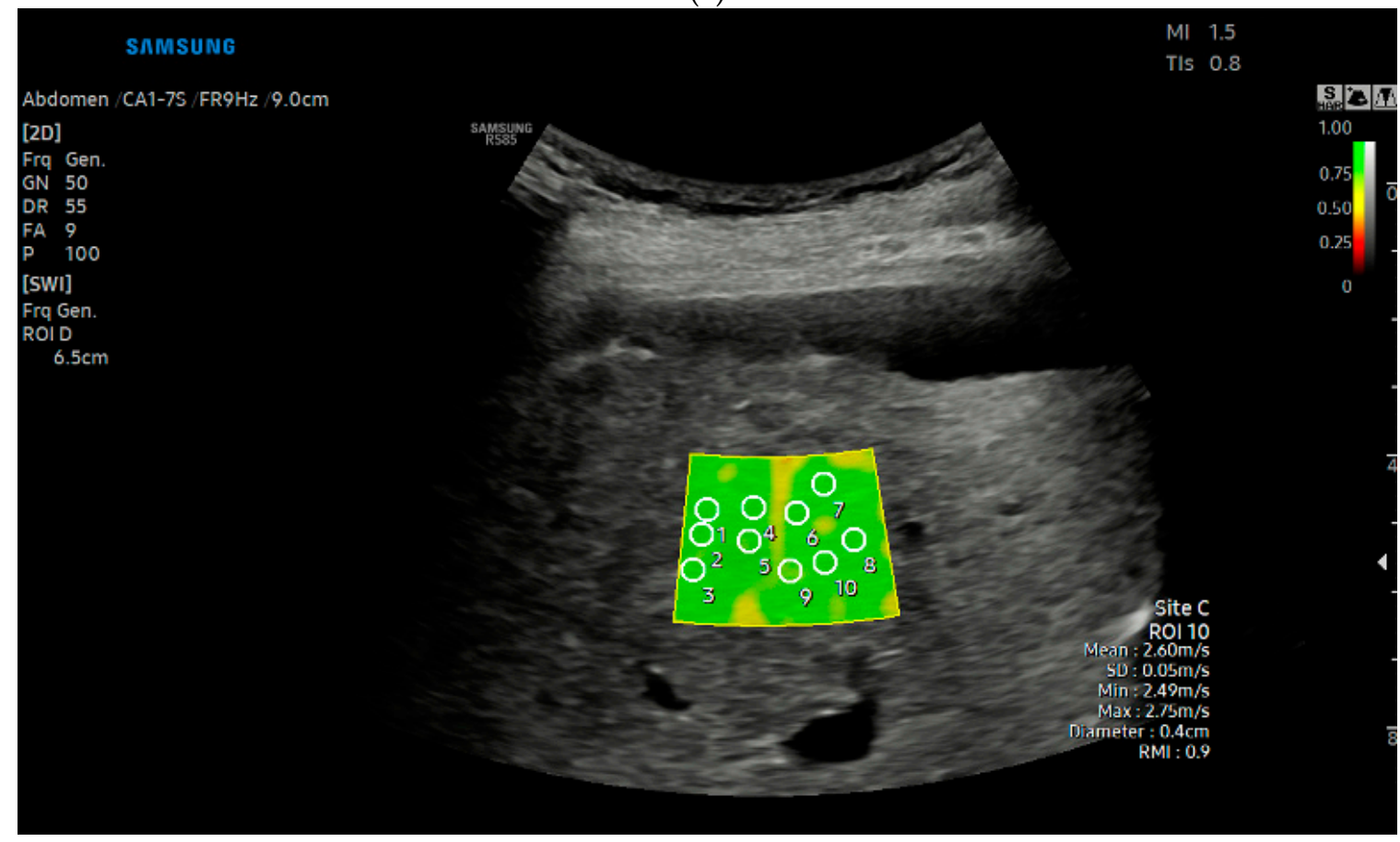

(b)

Figure 1. Liver stiffness (LS) measurement with 2D shear wave elastography (SWE). (a) LS measurement of a cirrhotic patient. The LS was reported in $\mathrm{m} / \mathrm{s}$ units. (b) Color-coded map of the reliable measure index (RMI) values of SWE measurement was used for ROI selection in the cirrhotic liver parenchyma. Only measurements with an RMI $\geq 0.4$ were considered acceptable. 


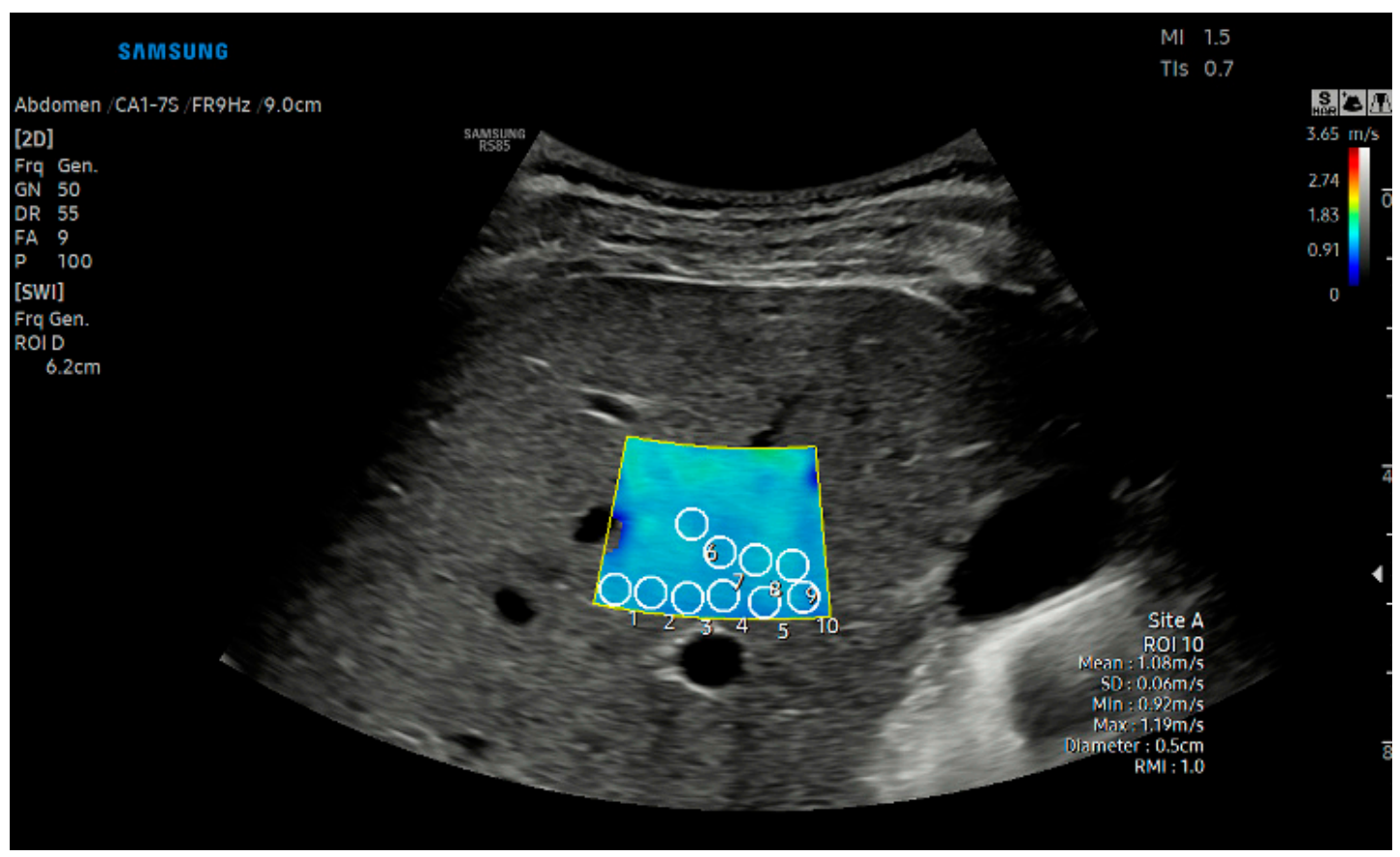

(a)

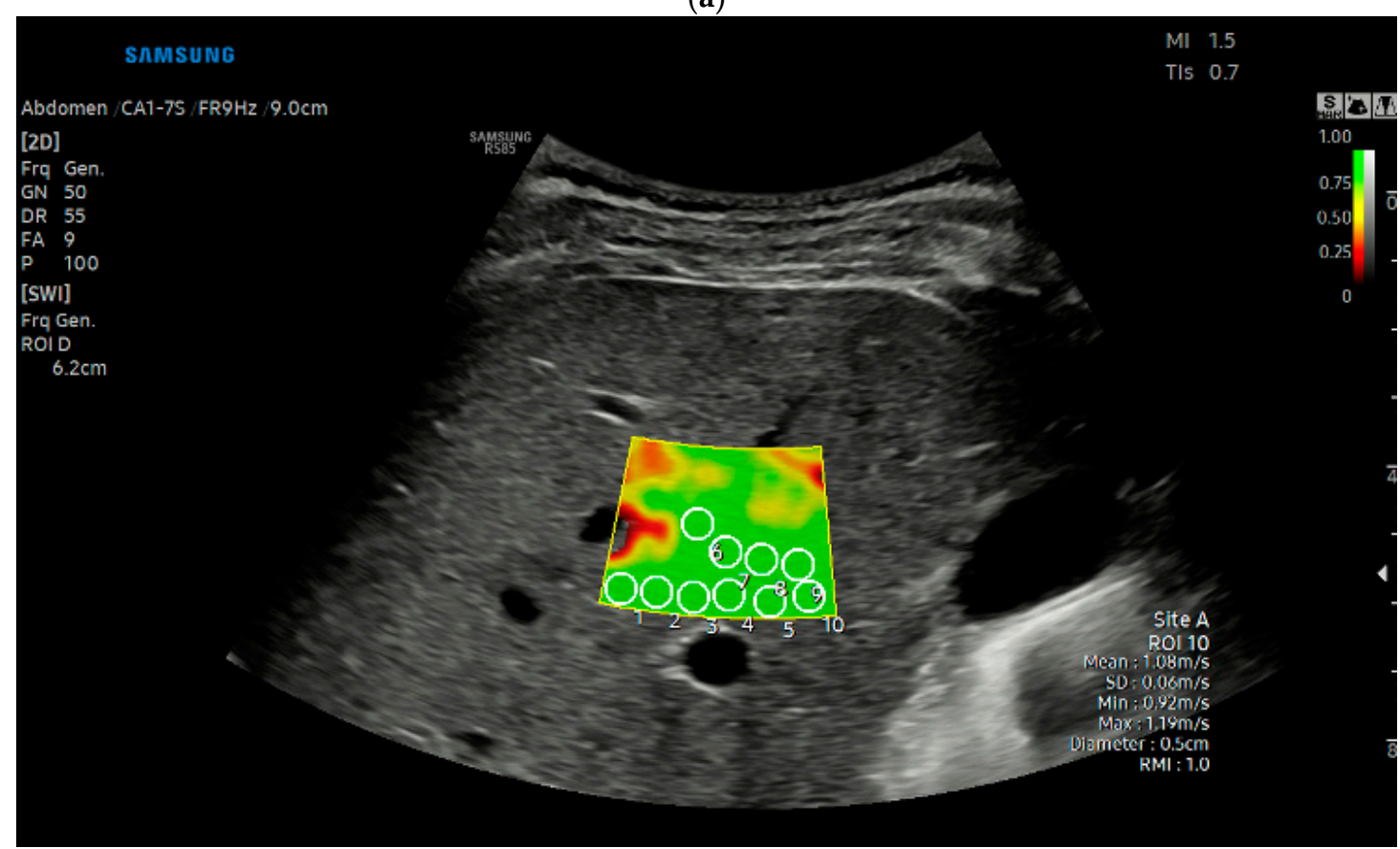

(b)

Figure 2. Liver stiffness (LS) measurement with 2D shear wave elastography (SWE). (a) SWE measurement of a liver with no significant fibrosis. The LS was reported in m/s units. (b) Color-coded map of the RMI was used for the ROI selection during 2D-SWE of a liver with no significant fibrosis. 


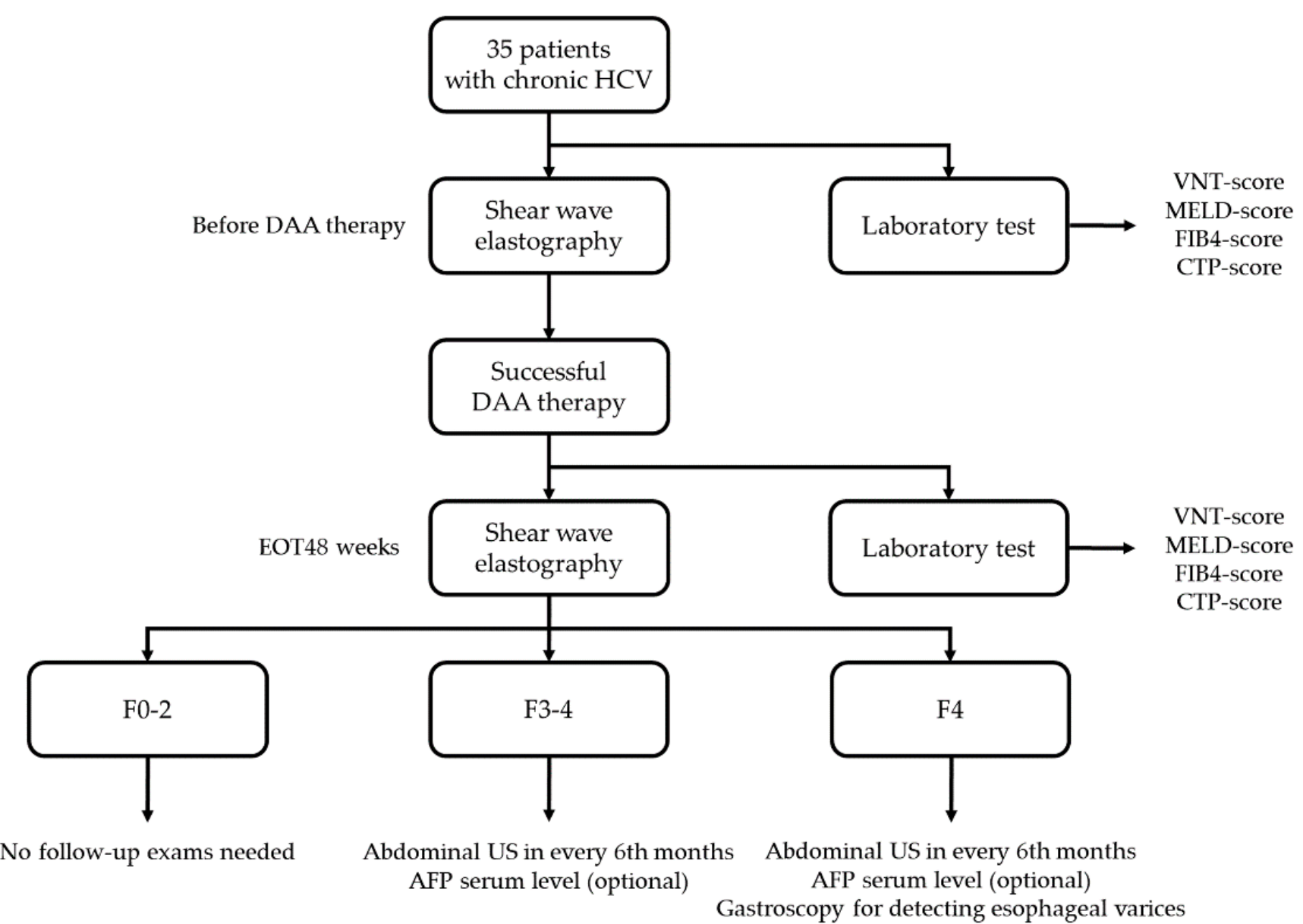

Figure 3. The flowchart shows the design of our prospective study. We prospectively enrolled 35 patients who were diagnosed with chronic hepatitis $C$ virus infection and who attained sustained virological response after treatment with DAA agents or interferon-based treatment. The METAVIR score of liver fibrosis was determined for each patient both before and after the antiviral treatment based on the result of the shear wave elastography measurement. The FIB4 score, the number of patients with varices needing treatment, the Child-Turcotte-Pugh class, and the MELD-score were calculated from laboratory tests as it was described previously [17,19-21]. HCV: hepatitis C virus; DAA: direct-acting antiviral therapy; EOT48w: 48 weeks after successful antiviral treatment; VNT: varices needing treatment; MELD: model for end-stage liver disease; FIB4-score: fibrosis-4 score; CTP: Child-Turcotte-Pugh; AFP: alpha-fetoprotein; US: ultrasound.

\subsection{Statistical Analysis}

The paired Student's $t$-test was used to compare pre- and post-treatment LS values and laboratory parameters. Fisher's exact test was used to compare the distributions of categorical variables such as cirrhosis stage and VNT risk before and after treatment. The odds ratios (OR) are reported together with the $95 \%$ confidence intervals (CI). In the case of continuous variables, we report the mean and the standard deviation (SD); in the case of categorical variables, the frequency and the percentage are reported. We set the limit of statistical significance at $p<0.05$. The statistical analysis was completed with the $\mathrm{R} \times 64$ v3.4.1 software package (www.r-project.org accessed on 12th March 2021).

\section{Results}

\subsection{LS Decreases after Successful Antiviral Therapy}

The liver stiffness was significantly lower $(p<0.001)$ at 48 weeks after EOT $(1.90 \pm 0.50 \mathrm{~m} / \mathrm{s})$ compared to the matched values before treatment $(2.59 \pm 0.89 \mathrm{~m} / \mathrm{s})$. The METAVIR score was calculated at each time point based on the SWE measurements, and it also showed significant improvement $(p<0.028)$ after treatment $(\mathrm{F} 0 / 1=9, \mathrm{~F} 2=2, \mathrm{~F} 3=10, \mathrm{~F} 4=14)$ 
compared to the baseline $(\mathrm{F} 0 / 1=2, \mathrm{~F} 2=1, \mathrm{~F} 3=7, \mathrm{~F} 4=25)$ (Figure 4) (Table 1). The number of cirrhotic cases (F4) was also significantly lower $(\mathrm{OR}=0.27,95 \% \mathrm{CI}=0.09-0.72, p<0.016)$ at EOT48w. The average decrease in LS was greater $(p<0.028)$ in cirrhotic cases $(\mathrm{F} 4, \Delta$-LS $=$ $-0.87 \pm 0.79 \mathrm{~m} / \mathrm{s}$ ) than in non-cirrhotic cases (F0/1, $\Delta \mathrm{LS}=-0.26 \pm 0.37)$. The LS improved in 31 out of 35 patients in SVR, and it slightly increased in only four cases, including two cirrhotic and two F2 grade fibrosis patients (Figure 5).

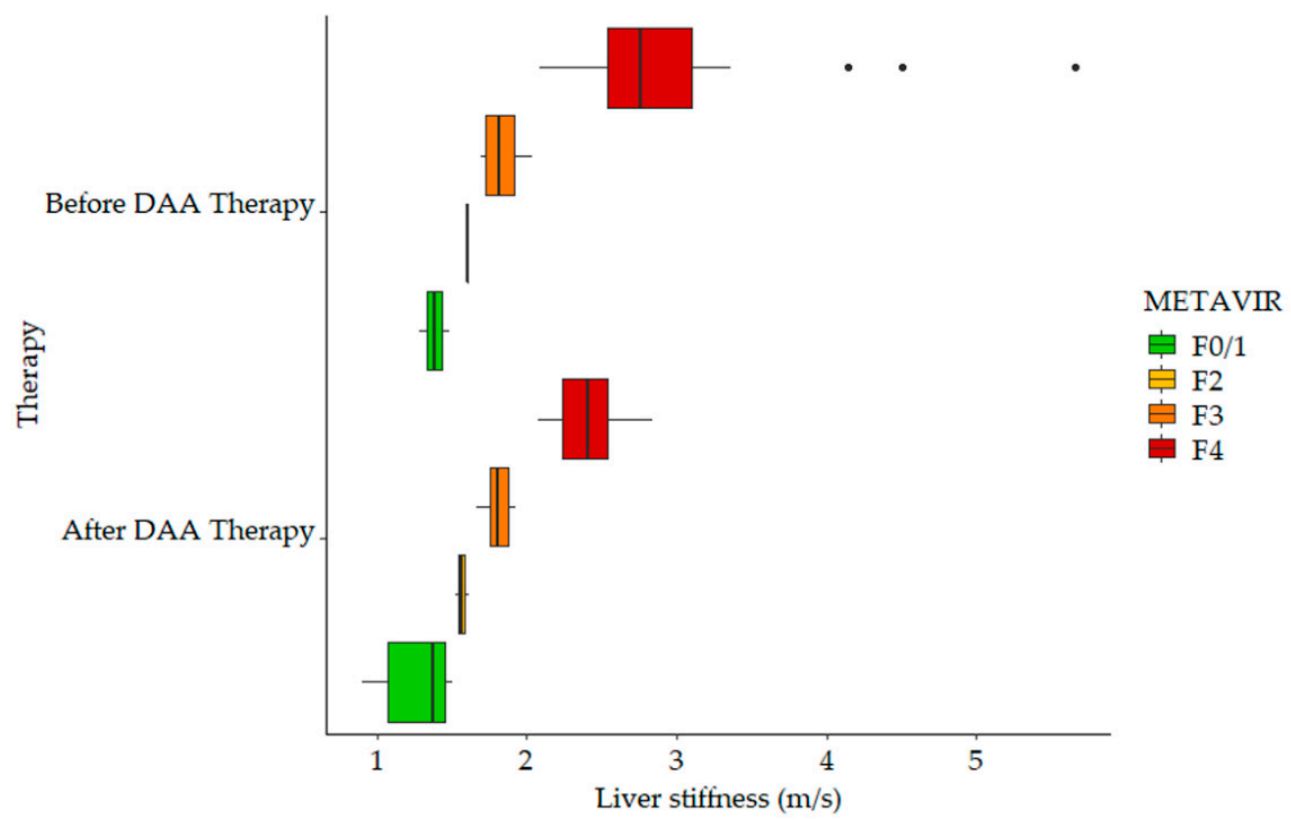

Figure 4. The boxplots demonstrate the improvement of liver fibrosis following antiviral treatment. Liver fibrosis was classified into four METAVIR stages (F0/1, F2, F3, F4) based on the SWE measurement. The post-treatment measurement was performed 48 weeks after the completion of antiviral treatment. We detected significantly improved fibrosis and a lower proportion of cirrhotic cases after successful direct-acting antiviral (DAA) therapy.

Table 1. Comparison between pre- and post-treatment liver stiffness and METAVIR scores.

\begin{tabular}{ccccc}
\hline & Baseline & EOT48w $^{\mathbf{1}}$ & $p$-Value $^{\mathbf{2}}$ & Odds-Ratio $^{\text {Odd }}$ \\
\hline LS & $2.59 \pm 0.89 \mathrm{~m} / \mathrm{s}$ & $1.90 \pm 0.50 \mathrm{~m} / \mathrm{s}$ & $p<0.001$ & NA \\
METAVIR $^{3}$ & & & $p<0.028$ & NA \\
F0/1 & $2(5.7 \%)$ & $9(25.7 \%)$ & $p<0.045$ & $5.71(1.13-28.74)$ \\
F2 & $1(2.8 \%)$ & $2(5.7 \%)$ & $p<1.00$ & $2.06(0.18-23.82)$ \\
F3 & $7(20 \%)$ & $10(28.6 \%)$ & $p<0.578$ & $1.60(0.53-4.83)$ \\
F4 & $25(71.4 \%)$ & $14(40 \%)$ & $p<0.015$ & $0.27(0.09-0.72)$ \\
\hline
\end{tabular}

\footnotetext{
${ }^{1} 48$ weeks after the completion of antiviral treatment with sustained virological response. ${ }^{2}$ For comparing means between pre- and post-treatment groups, the paired Student's $t$-test was used; for frequencies, the Fisher's exact test was used. ${ }^{3}$ The METAVIR score was calculated from liver stiffness by using the previously described cutoff values. LS: liver stiffness; NA: not applicable.
} 


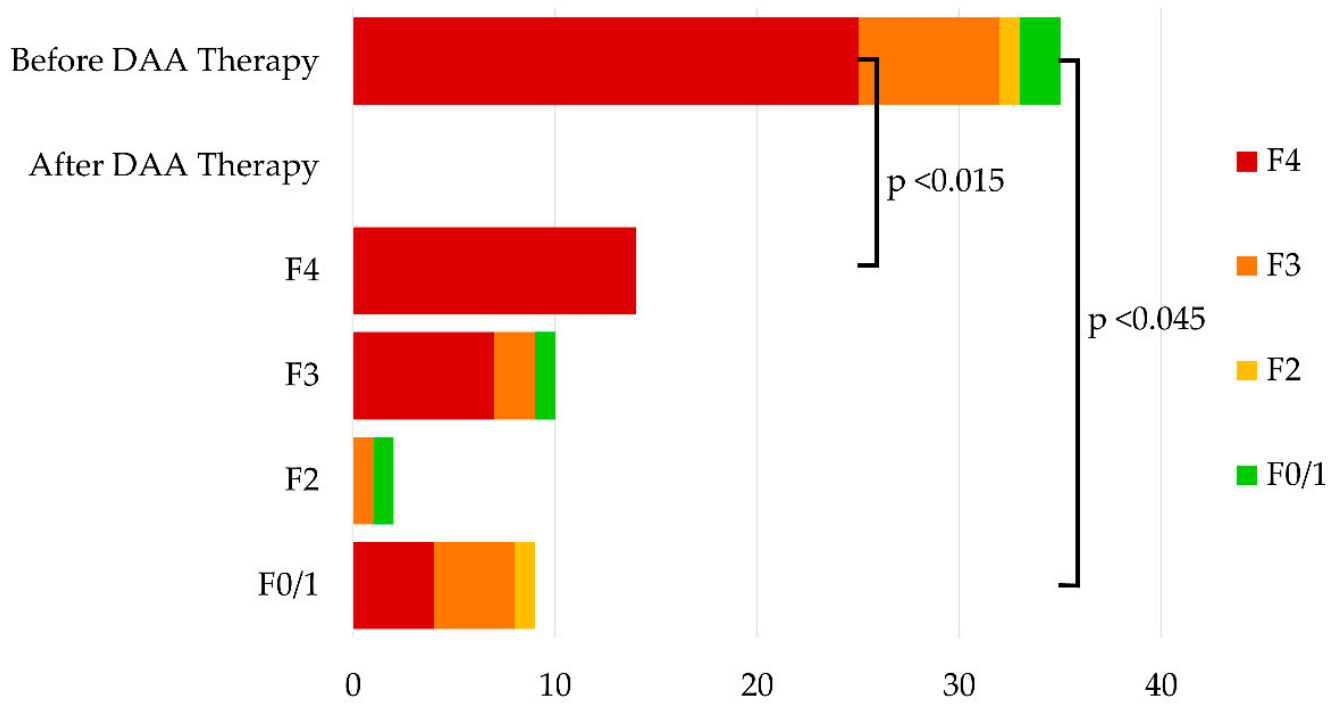

Figure 5. The bar plots demonstrate the improvement of liver fibrosis following antiviral treatment. The liver stiffness was determined during a pre-treatment ultrasound scan with shear wave elastography (SWE). Liver fibrosis was classified into four METAVIR stages (F0/1, F2, F3, F4) based on the SWE measurement. The post-treatment measurement was performed 48 weeks after the completion of antiviral treatment. The number of patients in the different METAVIR stages is represented by the colored bands of the bar plots. Based on the METAVIR score, the number of cirrhotic cases (F4) was significantly lover $(p<0.015)$ at EOT48w, while the number of cases with F0/1 showed significant increasement $(p<0.045)$ after treatment compared to the baseline.

\subsection{Laboratory Tests Improve in Parallel with LS}

The ALT level significantly improved after treatment, and it returned to the normal range with two exceptions in all patients at EOT48w. In comparison, only 10 patients had normal ALT at the baseline $(\mathrm{OR}=0.02,95 \% \mathrm{CI}=0.005-0.12, p<0.001)$. The GGT level was elevated in 26 patients before treatment but only in seven patients at the time of post-treatment follow-up $(\mathrm{OR}=0.087,95 \% \mathrm{CI}=0.03-0.27, p<0.001)$. The ALB returned to the normal range in all six cirrhotic patients who had low baseline levels. AST, TBILL, and CRE levels at EOT48w were also closer to the normal range than the pre-treatment levels. The rest of the serum markers, including PLT, INR, and $\mathrm{Na}$, all showed an improvement, but they did not reach statistical significance (Table 2). We calculated the FIB4 score as an alternative non-invasive laboratory marker of liver fibrosis. Similar to the SWE, the FIB4 score also indicated significantly $(p<0.018)$ lower post-treatment fibrosis $(2.04 \pm 1.12)$ compared to the pre-treatment status $(3.51 \pm 2.24)$.

Table 2. Comparison of laboratory tests before and after treatment.

\begin{tabular}{cccc}
\hline & Baseline & EOT48 $^{\mathbf{1}}$ & $p$-Value ${ }^{\mathbf{2}}$ \\
\hline AST $(\mathrm{IU} / \mathrm{L})$ & 75.7 & 27.7 & $p<0.019$ \\
ALT $(\mathrm{IU} / \mathrm{L})$ & 92.7 & 25 & $p<0.001$ \\
GGT $(\mathrm{IU} / \mathrm{L})$ & 137.1 & 54.1 & $p<0.001$ \\
TBILL $(\mu \mathrm{mol} / \mathrm{L})$ & 20.5 & 16.9 & $p<0.030$ \\
ALB $(\mathrm{g} / \mathrm{L})$ & 41.3 & 43.8 & $p<0.001$ \\
CRE $(\mu \mathrm{mol} / \mathrm{L})$ & 70.7 & 74 & $p<0.023$ \\
Na $(\mathrm{mmol} / \mathrm{L})$ & 138.1 & 139.6 & $p<0.104$ \\
INR & 1.2 & 1.3 & $p<0.288$ \\
PLT $\left(10^{9} / \mathrm{L}\right)$ & 168.5 & 180 & $p<0.249$ \\
\hline
\end{tabular}

${ }^{1} 48$ weeks after the completion of antiviral treatment with sustained virological response. ${ }^{2}$ For comparing means between pre- and post-treatment groups, the paired Student's $t$-test was used, while for frequencies, the Fisher's exact test was used. ALB: serum albumin, ALT: alanine aminotransferase, AST: aspartate aminotransferase, CRE: serum creatinine, GGT: gamma-glutamyl transpeptidase, INR: international normalized ratio, Na: serum sodium, PLT: platelet, count, TBILL: total bilirubin. 


\subsection{The Risk of Esophageal VNT Decreases in SVR}

We also calculated the CTP and the MELD scores for each patient to assess clinical improvement following antiviral therapy (Table 3). Five CTP class B cirrhotic patients were identified in the original patient cohort, and four of these converted to class A after treatment. A small to moderate amount of ascites was observed in two patients at the baseline and in one patient at EOT48w on the ultrasound scan. The MELD score did not change significantly; out of two patients with high mortality risk (MELD > 20) before treatment, only one had lower risk (MELD $\leq 20)$ at EOT48w (Table 3).

Table 3. Changes in clinical risk factors due to anti-HCV therapy.

\begin{tabular}{ccccc}
\hline & Baseline & EOT48w $^{\mathbf{1}}$ & $p$-Value & Odds Ratio \\
\hline Ascites & $2(6 \%)$ & $1(3 \%)$ & $p<1.00$ & $0.49(0.04-5.61)$ \\
FIB4 score $^{3}$ & $3.51 \pm 2.24$ & $2.04 \pm 1.12$ & $p<0.018$ & NA \\
VNT $^{3}$ & $15(42 \%)$ & $4(11 \%)$ & $p<0.007$ & $0.17(0.05-0.59)$ \\
CTP class $^{3}$ & & & & \\
A & $30(86 \%)$ & $34(97 \%)$ & $p<0.127$ & $5.67(0.63-51.27)$ \\
B & $5(14 \%)$ & $1(3 \%)$ & & $0.18(0.02-1.60)$ \\
C & 0 & 0 & NA & NA \\
MELD score & & & & \\
$<10$ & $25(71 \%)$ & $25(71 \%)$ & $p<1.00$ & $1.00(0.35-2.82)$ \\
$<20$ & $8(23 \%)$ & $9(26 \%)$ & $p<1.00$ & $0.86(0.29-2.56)$ \\
$<25$ & $2(6 \%)$ & $1(3 \%)$ & $p<0.619$ & $2.06(0.18-23.82)$ \\
$\geq 25$ & 0 & 0 & NA & NA \\
\hline
\end{tabular}

${ }^{1} 48$ weeks after the completion of antiviral treatment with sustained virological response. ${ }^{2}$ For comparing means between pre- and post-treatment groups, the paired Student's $t$-test was used; for frequencies, the Fisher's exact test was used. ${ }^{3}$ The FIB4 score, the number of patients with VNT, the CTP class, and the MELD score were calculated as described previously [17,19-21]. CTP: Child-Turcott-Pugh classification, Fib-4: fibrosis-4 score, MELD: Model for End-Stage Liver Disease, VNT: varices needing treatment.

The risk of VNT was calculated from the PLT and the LS values according to the Baveno VI guidelines. High-risk of esophageal VNT was detected in 15 cirrhotic patients at baseline and in four patients at the follow-up ( $\mathrm{OR}=0.17,95 \% \mathrm{CI}=0.05-0.59, p<0.007)$.

\section{Discussion}

Because the stage of liver fibrosis is a key predictor of complications and mortality in patients with chronic HCV infection, we aimed to measure liver fibrosis with SWE before and after SVR in patients treated mainly with DAAs. According to public databases, there is an increasing number of chronic hepatitis $C$ patients with advanced liver fibrosis who became virus-free as a result of modern antiviral therapy [22]. If reversal of fibrosis could be demonstrated with non-invasive techniques, such as SWE, it could result in the reclassification of previously high-risk patients to a lower risk category of liver diseaserelated complications; eventually, the reassessment with SWE would alter the management and the long-term outcome of these patients.

D'Ambrosio and colleagues published an article in 2012 summarizing the long-term follow-up of 38 chronic hepatitis C patients after reaching SVR [23]. Their prospective study included cirrhotic patients who received successful peg-IFN and ribavirin therapy, attained SVR, and they were followed for at least four years afterward. Semiquantitative histological staging of fibrosis according to the METAVIR system as well as a morphometric determination of collagen content, assessment of necroinflammatory activity, and ductular proliferation were performed on paired pre- and post-treatment liver biopsies. All of the 38 enrolled patients had F4 stage fibrosis pre-treatment according to the METAVIR staging system. As the result of the treatment, the fibrosis in $61 \%$ of the patients was categorized as at least one stage lower at an average follow-up of 61 months from SVR. In the samples of post-treatment F4 patients, the fibrotic area was also significantly reduced based on the collagen content compared to the pre-treatment samples. Our result corroborates the above findings as we also observed down-staging of cirrhosis (F4) in $46 \%$ and advanced 
fibrosis (F3) in an additional 30\% of the cases at 48 weeks after EOT based on the SWE. Additionally, we measured lower LS with two exceptions in all patients, including even those who did not change into a lower METAVIR stage. It is important to emphasize that biopsy specimens demonstrated an $89 \%$ reduction in collagen content, which could explain the lower LS values in SVR, as LS observed with SWE were clearly shown to be in direct correlation with the amount of fibrosis measured on biopsy [24]. Meanwhile, our follow-up time was much shorter, 12 months vs. 61 months in SVR; thus, the dissolution of the necroinflammatory infiltrates might have contributed to the relatively rapid improvement in LS. Examination of serum AST levels found that 37 patients initially had elevated levels, while 36 patients had AST levels in the normal range at the end of treatment [23]. We also observed a similar trend in our patient population where AST levels returned to the normal range with two exceptions and ALT with one exception in all patients. Similar findings were reported by Tachi et al., who followed 176 INF and DAA-treated patients in SVR with ARFI in a prospective study [25]. The authors also compared the elastography results with another patient cohort of 140 patients who had paired pre- and post-treatment liver biopsies. There was a significant reduction in LS at 24 weeks after EOT compared to the baseline LS in all stages. The highest drop in LS was detected in the F3 stage. The grade of inflammatory activity on the pre-treatment biopsy was also a significant independent predictor of post-treatment LS reduction in a multivariate linear regression analysis. Based on their observations, the authors concluded that, in addition to slow improvement in liver fibrosis, resolution of the inflammation also contributes to short-term improvement in LS after EOT.

Facciorusso et al. examined the long-term effects of antiviral therapy on liver fibrosis in 153 patients using TE [26]. Of their patients, 70 received interferon-based treatment, and 83 received DAA therapy. Initially, 34.6\% of their patients were diagnosed with cirrhosis. In total, 112 of their patients attained SVR, and 41 were found to be non-responders. Using $12.5 \mathrm{kPa}$ as the cutoff value, $32.1 \%$ of patients were classified as METAVIR stage F4 before therapy, while $87.5 \%$ were classified as F3. The number of patients with F4-stage disease decreased significantly at the end of treatment to only $20.5 \%$. The authors observed that the mean LS of patients achieving SVR decreased from $12.3 \mathrm{kPa}$ to $6.6 \mathrm{kPa}$ by the end of the 5 year follow-up. The largest decrease was observed in the early follow-up period, which was $2.5 \mathrm{kPa}$ at EOT and $3.7 \mathrm{kPa}$ at six months after EOT. After the first year, the rate of LS decline slowed down. The number of cirrhotic patients was halved at six months, and it was less than 5\% at four years after EOT. Thus, it was demonstrated that LS significantly decreases after SVR is attained, and the rate of decline is the highest during the first year after EOT. Our results are consistent with the above-described observations. However, in our study, we used SWE, our patients were treated mostly with DAAs, and all of them were in SVR at the follow-up at 48 weeks EOT. These differences in the study design may explain why we saw a higher drop in LS, from $2.59 \mathrm{~m} / \mathrm{s}(20.5 \mathrm{kPa})$ to $1.90 \mathrm{~m} / \mathrm{s}(11.7 \mathrm{kPa})$, in our patient cohort at one year from EOT.

Attia et al., who used TE and ARFI elastography to follow up with 275 chronic hepatitis $C$ patients after successful DAA treatment, also detected a significant decrease (from $2.04 \mathrm{~m} / \mathrm{s}$ to $1.75 \mathrm{~m} / \mathrm{s}$ ) in LS at 24 weeks after EOT with both techniques [15]. Both the incidence and the rate of the decline in LS were higher in cirrhotic patients attaining SVR than in patients with lower-grade fibrosis. Meanwhile, the LS of patients with advancedstage decompensated liver disease at baseline was more likely to progress, even during SVR. In all the patients whose LS progressed during the follow-up, the baseline TE measurement was above $17 \mathrm{kPa}$, which is associated with clinically significant portal hypertension according to the Baveno VI guidelines. According to the authors, these findings suggest that, in patients with decompensated disease and portal hypertension, the fibrosis reaches a stage where the clearance of HCV cannot reverse the progression. In comparison, our patient cohort included 15 patients with clinically significant hypertension and VNT; however, we found progression in LS in only two of these patients (13\%), which indicates improving fibrosis in the majority of the decompensated patients after clearance of $\mathrm{HCV}$. 
Therefore, our findings suggest that even patients with advanced-stage fibrosis need efficient antiviral treatment, as it could lower the risk of severe complications.

Clinical studies also demonstrated that SVR is associated with a lower risk of variceal bleeding $[27,28]$. The Baveno VI Consensus Workshop guideline recommends stratification of patients with a cutoff at LS $>20 \mathrm{kP}$ and PLT $<150,000 \mu / \mathrm{L}$ to identify the group with a high risk of clinically significant varices. However, the utility of the Baveno VI criteria is not yet evaluated in SVR. We found a reduced risk of esophageal VNT according to the Baveno VI guidelines in $73 \%$ of the high-risk patients who achieved SVR. Our study is among the first to demonstrate that SWE is able to determine the lower risk of variceal bleeding post-SVR, which may simplify the clinical management of these patients. According to the American Gastroenterological Association Institute, it is recommended that HCVRNA levels be measured 12 to 48 weeks after EOT, and testing 24 weeks after EOT is recommended only in select cases [16]. According to the directive, routine RNA testing after confirmation of viral freedom by PCR is only recommended for patients with a high risk of re-infection, as other patients have a low probability of late relapse, thus, if their complaint or symptoms do not make it necessary, further RNA detection-based screening is not required. Endoscopic varicose screening is also recommended for all patients with cirrhosis, the outcome of which (rather than the attainment of SVR) necessitates further testing and follow-up [16]. An important objective of the continued follow-ups in SVR is screening for HCC, which is not required in people with stage $0-2$ fibrosis. However, in patients who had at least stage 3 fibrosis initially, HCC screening should be continued, even after viral clearance, using a combination of imaging and AFP measurement twice a year. The screening should be continued for the rest of the life of the affected patients, because the incidence of HCC may remain higher in patients with advanced fibrosis, even a long time after viral clearance is achieved [25,29]. This is especially true for those patients who consume alcohol frequently. Our results indicate that a complementary SWE can improve the prognostic value of regular ultrasound screening by identifying patients with resolving fibrosis, who, therefore, may have a lower risk of HCC and may require less frequent follow-ups, although multi-center longitudinal studies are still required to determine long-term changes in LS post-SVR as well as their effect on the incidence of HCC.

\section{Conclusions}

In summary, SVR in chronic hepatitis $C$ is associated with significantly more favorable outcomes and a lower risk of serious complications. Nevertheless, the chance of developing HCC or variceal bleeding still remains higher than in the general population. Therefore, close monitoring with imaging and clinical studies is recommended, especially for patients with advanced-stage pre-treatment fibrosis. Our study demonstrates a significant reduction in LS and consequently in the non-invasive staging of fibrosis a year after SVR is attained. These results may indicate that adding SWE to the follow-up imaging protocol can improve patient management by amending the initial assessment of the fibrosis stage and the identification of patients whose risk of liver disease-related complications is significantly lowered as the result of SVR. Thus, SWE can contribute to the development of more efficient follow-up protocols which are better tailored to the individual needs of chronic hepatitis $C$ patients with liver fibrosis.

Author Contributions: A.F. and P.N.K. were responsible for the conception and design of the study; P.N.K. and G.G. performed the liver ultrasound and elastography measurements; B.K.B., A.D.R., P.B. and V.O. contributed to data collection and analysis; A.F. and F.S. contributed to patient selection and collection of clinical data; B.K.B. and V.O. contributed to editing and reviewing the manuscript. All authors have read and agreed to the published version of the manuscript.

Funding: P.N.K. (Bolyai 386/2017) was supported by the János Bolyai Research Scholarship of the Hungarian Academy of Sciences (https:/ / mta.hu/bolyai-osztondij accessed on 23rd April 2021). B.K.B. and P.N.K. received financial support from the GINOP 2-2-18 grant. The funders had no role in the study design, data collection and analysis, decision to publish, or preparation of the manuscript. 
Institutional Review Board Statement: The study was conducted according to the guidelines of the Declaration of Helsinki, and approved by the Ethics Committee of the Semmelweis University (Semmelweis University Regional and Institutional Committee of Science and Research) (SE-TUKEB 163/2017, date of approval: 7 August 2017).

Informed Consent Statement: Informed consent was obtained from all subjects involved in the study.

Data Availability Statement: The data presented in this study are available on request from the corresponding author.

Acknowledgments: The authors would like to express their gratitude to Sonarmed Ltd. and to Samsung Ultrasound Training Center at Semmelweis University for providing an ultrasound scanner for the study. The authors thank László Giay for his enthusiastic support of our research. We are also thankful to Zsuzsa Kizman from the 1st Department of Internal Medicine for her assistance with patient appointments and data collection.

Conflicts of Interest: The authors declare no conflict of interest.

\section{References}

1. Lombardi, A.; Mondelli, M.U. ESCMID Study Group for Viral Hepatitis (ESGVH) Hepatitis C: Is eradication possible? Liver Int. 2019, 39, 416-426. [CrossRef]

2. Pawlotsky, J.-M.; Negro, F.; Aghemo, A.; Berenguer, M.; Dalgard, O.; Dusheiko, G.; Marra, F.; Puoti, M.; Wedemeyer, H. EASL Recommendations on Treatment of Hepatitis C. J. Hepatol. 2018, 69, 461-511. [CrossRef]

3. Holmes, A.J.; Rutledge, S.M.; Chung, R.T. Direct-Acting Antiviral Treatment for Hepatitis C. Lancet 2019, 393, 1392-1394. [CrossRef]

4. Ghany, M.G.; Morgan, T.R.; Panel, A.H.C.G.; Marks, K.M.; Wyles, D.L.; Aronsohn, A.I.; Bhattacharya, D.; Broder, T.; FaladeNwulia, O.O.; Feld, J.J.; et al. Hepatitis C Guidance 2019 Update: American Association for the Study of Liver Diseases-Infectious Diseases Society of America Recommendations for Testing, Managing, and Treating Hepatitis C Virus Infection. Hepatology 2019, 71, 686-721. [CrossRef] [PubMed]

5. Budai, B.K.; Tóth, A.; Borsos, P.; Frank, V.G.; Shariati, S.; Fejér, B.; Folhoffer, A.; Szalay, F.; Bérczi, V.; Kaposi, P.N. ThreeDimensional CT Texture Analysis of Anatomic Liver Segments can Differentiate between Low-Grade and High-Grade Fibrosis. BMC Med Imaging 2020, 20, 1-11. [CrossRef] [PubMed]

6. Low, G. General Review of Magnetic Resonance Elastography. World J. Radiol. 2016, 8, 59-72. [CrossRef]

7. Ferraioli, G.; Tinelli, C.; Bello, B.D.; Zicchetti, M.; Filice, G.; Filice, C.; Liver Fibrosis Study Group. Accuracy of Real-Time Shear Wave Elastography for Assessing Liver Fibrosis in Chronic Hepatitis C: A Pilot Study. Hepatology 2012, 56, 2125-2133. [CrossRef]

8. Karlas, T.; Pfrepper, C.; Wiegand, J.; Wittekind, C.; Neuschulz, M.; Mössner, J.; Berg, T.; Tröltzsch, M.; Keim, V. Acoustic Radiation Force Impulse Imaging (ARFI) for Non-Invasive Detection of Liver Fibrosis: Examination Standards and Evaluation of Interlobe Differences in Healthy Subjects and Chronic Liver Disease. Scand. J. Gastroenterol. 2011, 46, 1458-1467. [CrossRef] [PubMed]

9. Bota, S.; Herkner, H.; Sporea, I.; Salzl, P.; Sirli, R.; Neghina, A.M.; Peck-Radosavljevic, M. Meta-Analysis: ARFI Elastography Versus Transient Elastography for the Evaluation of Liver Fibrosis. Liver Int. 2013, 33, 1138-1147. [CrossRef]

10. Kaposi, P.N.; Unger, Z.; Fejér, B.; Kucsa, A.; Tóth, A.; Folhoffer, A.; Szalay, F.; Bérczi, V. Interobserver Agreement and Diagnostic Accuracy of Shearwave Elastography for the Staging of Hepatitis C Virus-Associated Liver Fibrosis. J. Clin. Ultrasound 2019, 48, 67-74. [CrossRef] [PubMed]

11. European Association for the Study of the Liver; Asociación Latinoamericana Para el Estudio Del Hígado. EASL-ALEH Clinical Practice Guidelines: Non-Invasive Tests for Evaluation of Liver Disease Severity and Prognosis. J. Hepatol. 2015, 63, 237-264. [CrossRef] [PubMed]

12. Chekuri, S.; Nickerson, J.; Bichoupan, K.; Sefcik, R.; Doobay, K.; Chang, S.; DelBello, D.; Harty, A.; Dieterich, U.T.; Perumalswami, P.V.; et al. Liver Stiffness Decreases Rapidly in Response to Successful Hepatitis C Treatment and Then Plateaus. PLOS ONE 2016, 11, e0159413. [CrossRef]

13. Hézode, C.; Castéra, L.; Roudot-Thoraval, F.; Bouvier-Alias, M.; Rosa, I.; Roulot, D.; Leroy, V.; Mallat, A.; Pawlotsky, J.-M. Liver Stiffness Diminishes with Antiviral Response in Chronic Hepatitis C. Aliment. Pharmacol. Ther. 2011, 34, 656-663. [CrossRef] [PubMed]

14. Libânio, D.; Marinho, R.T. Impact of Hepatitis C Oral Therapy in Portal Hypertension. World J. Gastroenterol. 2017, 23, 4669-4674. [CrossRef] [PubMed]

15. Attia, D.; Deterding, K.; Cornberg, J.; Gebel, M.J.; Cornberg, M.; Manns, M.P.; Wedemeyer, H.; Potthoff, A. Different Kinetics of Liver Stiffness using Shear Wave Elastography in Patients with Chronic Hepatitis C Infection Treated with Interferon-Free Regimens. Eur. J. Gastroenterol. Hepatol. 2019, 31, 67-74. [CrossRef] [PubMed]

16. Jacobson, I.M.; Lim, J.K.; Fried, M.W. American Gastroenterological Association Institute Clinical Practice Update-Expert Review: Care of Patients Who Have Achieved a Sustained Virologic Response After Antiviral Therapy for Chronic Hepatitis C Infection. Gastroenterology 2017, 152, 1578-1587. [CrossRef]

17. De Franchis, R. Expanding Consensus in Portal Hypertension. J. Hepatol. 2015, 63, 743-752. [CrossRef] 
18. Paternostro, R.; Reiberger, T.; Bucsics, T. Elastography-Based Screening for Esophageal Varices in Patients with Advanced Chronic Liver Disease. World J. Gastroenterol. 2019, 25, 308-329. [CrossRef] [PubMed]

19. Kamath, P.S.; Kim, W.R. The Model for End-Stage Liver Disease (MELD) \& Dagger. Hepatology 2007, 45, 797-805. [CrossRef]

20. Sterling, R.K.; Lissen, E.; Clumeck, N.; Sola, R.; Correa, M.C.; Montaner, J.; Sulkowski, M.S.; Torriani, F.J.; Dieterich, D.T.; Thomas, D.L.; et al. Development of a Simple Noninvasive Index to Predict Significant Fibrosis in Patients with HIV/HCV Coinfection. Hepatology 2006, 43, 1317-1325. [CrossRef] [PubMed]

21. Pugh, R.N.H.; Murray-Lyon, I.M.; Dawson, J.L.; Pietroni, M.C.; Williams, R. Transection of the Oesophagus for Bleeding Oesophageal Varices. BJS 2005, 60, 646-649. [CrossRef]

22. Marshall, A.D.; Pawlotsky, J.-M.; Lazarus, J.V.; Aghemo, A.; Dore, G.J.; Grebely, J. The Removal of DAA Restrictions in Europe —One Step Closer to Eliminating HCV as a Major Public Health Threat. J. Hepatol. 2018, 69, 1188-1196. [CrossRef] [PubMed]

23. D'Ambrosio, R.; Aghemo, A.; Rumi, M.G.; Ronchi, G.; Donato, M.F.; Paradis, V.; Colombo, M.; Bedossa, P. A Morphometric and Immunohistochemical Study to Assess the Benefit of a Sustained Virological Response in Hepatitis C Virus Patients with Cirrhosis. Hepatology 2012, 56, 532-543. [CrossRef] [PubMed]

24. Ferraioli, G.; Tinelli, C.; Lissandrin, R.; Zicchetti, M.; Bello, B.D.; Filice, G.; Filice, C. Point Shear Wave Elastography Method for Assessing Liver Stiffness. World J. Gastroenterol. 2014, 20, 4787-4796. [CrossRef] [PubMed]

25. Tachi, Y.; Hirai, T.; Kojima, Y.; Ishizu, Y.; Honda, T.; Kuzuya, T.; Hayashi, K.; Ishigami, M.; Goto, H. Liver Stiffness Reduction Correlates with Histological Characteristics of Hepatitis C Patients with Sustained Virological Response. Liver Int. 2017, 38 , 59-67. [CrossRef]

26. Facciorusso, A.; Del Prete, V.; Turco, A.; Buccino, R.V.; Nacchiero, M.C.; Muscatiello, N. Long-Term Liver Stiffness Assessment in Hepatitis C Virus Patients Undergoing Antiviral Therapy: Results From a 5-year Cohort Study. J. Gastroenterol. Hepatol. 2018, 33, 942-949. [CrossRef]

27. Bruno, S.; Crosignani, A.; Facciotto, C.; Rossi, S.; Roffi, L.; Redaelli, A.; De Franchis, R.; Almasio, P.L.; Maisonneuve, P. Sustained Virologic Response Prevents the Development of Esophageal Varices in Compensated, Child-Pugh Class A Hepatitis C VirusInduced Cirrhosis. A 12-Year Prospective Follow-Up Study. Hepatology 2010, 51, 2069-2076. [CrossRef]

28. Mallet, V.; Gilgenkrantz, H.; Serpaggi, J.; Verkarre, V.; Vallet-Pichard, A.; Fontaine, H.; Pol, S. Brief Communication: The Relationship of Regression of Cirrhosis to Outcome in Chronic Hepatitis C. Ann. Intern. Med. 2008, 149, 399-403. [CrossRef]

29. Nagaoki, Y.; Aikata, H.; Nakano, N.; Shinohara, F.; Nakamura, Y.; Hatooka, M.; Morio, K.; Kan, H.; Fujino, H.; Kobayashi, T.; et al. Development of Hepatocellular Carcinoma in Patients with Hepatitis C Virus Infection Who Achieved Sustained Virological Response Following Interferon Therapy: A Large-Scale, Long-Term Cohort Study. J. Gastroenterol. Hepatol. 2015, 31, 1009-1015. [CrossRef] [PubMed] 